\title{
Gliding Movements of Microtubule Driven by Kinesin Motors under External Force: Steering and Detachment
}

\author{
Takahiro Nitta \\ Gifu University \\ 1-1 Yanagido \\ Gifu, 501-1193, Japan \\ $+81-58-293-2551$ \\ nittat@gifu-u.ac.jp
}

\author{
Kouki Kawauchi \\ Gifu University \\ 1-1 Yanagido \\ Gifu, 501-1193, Japan \\ r3029010@edu.gifu-u.ac.jp
}

\begin{abstract}
Kinesin and microtubule have been utilized for applications, such as molecular communication. In this paper, we describe a computer simulation which reproduced movements of microtubules gliding over kinesin-coated glass surfaces under external force. The simulation qualitatively reproduced experimental results of microtubule movements under external force. This simulation would enable understanding of details of the gliding movements of microtubules under external force.
\end{abstract}

\section{Keywords}

Kinesin, microtubule, gliding assay, simulation, external control

\section{INTRODUCTION}

Biological molecular machines are attractive for nanotechnology, due to their small size and efficient conversion from chemical to mechanical energy. In particular, linear motor proteins, such as kinesin and myosin, have been utilized in synthetic environments for various applications [1], such as molecular communications [2] [3] [4]. The most commonly used implementation of the linear motor proteins is modified gliding assay. Gliding assay is a biophysical tool to investigate properties of motor proteins, where cytoskeletal filaments are propelled by their associated motor proteins coated on substrates. In order to utilize the gliding assay for applications, such as molecular communications and biosensors, cytoskeletal filaments are modified to bind information molecules or cargo.

One of important problems in utilizing the modified gliding assay is how to control the gliding movements. Although use of microfabricated substrates enables guiding of gliding movements of the cytoskeletal filaments, the guiding is passive. That is, dynamic operations, such as sorting, cannot be achieved. Applications of external forces from fluid flow, electric field and magnetic field have been utilized to steer the gliding movements of cytoskeletal filaments, enabling the dynamic operation. However, applications of external forces required tuning of their strength. Applying a stronger force leads to better control of their gliding movements, but leads to more cytoskeletal filaments to be detached from the surface [5].

Prediction of the optimal force density, defined as force acting on a segment of microtubules with the length of $1 \mu \mathrm{m}$, is difficult, because details of detachments are not known, and cannot be easily elucidated through experiments and analytical models, due to lack of detailed information, such as how individual motor protein binds to the associated cytoskeletons. Such information is difficult to obtain through experiments. And, even if such information was available, the system, which consists of hundreds of motor proteins interacting with a cytoskeletal filament, would be hard to deal with analytically.

Computer simulations can provide such information and make predictions, complementing experimental and theoretical studies. We have previously developed a Monte-Carlo simulation which reproduced gliding movements of microtubules under external forces [6]. In this simulation, we assumed that microtubules follow their leading tips, and that the microtubules were not detached from the surface. Although these assumptions hold at low external force, the simulation cannot be applied at high external force. Namely, sidewise slipping and detachments of microtubules cannot be simulated. In order to simulate the sidewise slipping and detachments, microtubules and kinesins have to be explicitly modeled. We have recently developed a simulation which explicitly models cytoskeletal filaments and motor proteins [7] [8]. In this study, we extended the simulation to include applied forces, and investigated whether the extended simulation reproduced experimental results.

\section{SIMULATION METHOD}

The simulation method was based on our previous work [7], and extended to include applied force to microtubules. In the following, we briefly summarize the simulation method. Microtubules were modeled as inextensible elastic rods with the flexural rigidity of $22.0 \mathrm{pN} \cdot \mu \mathrm{m}^{2}$. Time evolutions of conformations of the microtubules were computed with a Brownian dynamics simulation under constraints of fixed segment length. A uniform force field, corresponding to that from electric field, was applied to each segment of the microtubules. The strength of the force field will be described below. Kinesin motors were modeled as linear springs with the spring constant of 100 $\mathrm{pN} / \mu \mathrm{m}$. Once bound, kinesin heads were assumed to move toward microtubule plus ends. Hence, microtubules were propelled with the heading of their minus ends.

Simulation results were visualized with ParaView (http://www.paraview.org/). 


\section{RESULTS AND DISCUSSION}

Figure 1 shows a representative gliding movement of a microtubule. The surface density of kinesin was $10 \mu \mathrm{m}^{-2}$. The applied force density was $1.25 \mathrm{pN} / \mu \mathrm{m}$. The gliding movement was smooth, and the microtubule followed its leading tip. The trajectory roughly followed an arc. The radius of the curvatures of the arcs decreased when increasing the force density. This simulation result shows qualitative agreement with experiments [9] [10].

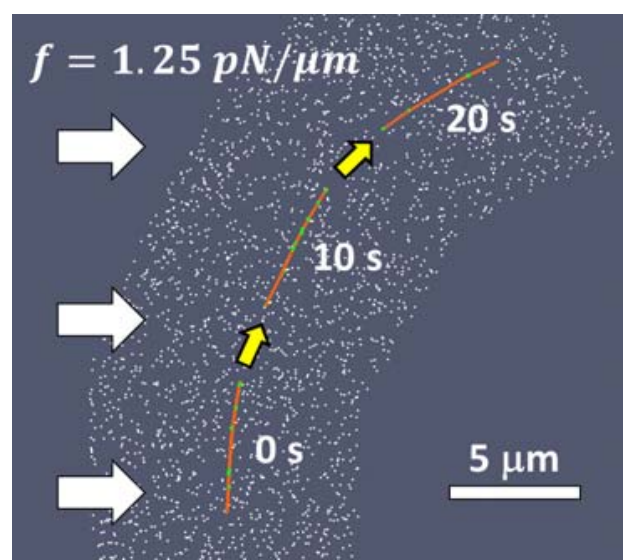

Figure 1. A representative gliding movement of microtubule under a uniform external force. The orange lines represent microtubules at 0,10 and $20 \mathrm{~s}$. Green dots represent kinesins attaching to the microtubules. White dots represent kinesins. The external force was applied from the right. The surface density of kinesins was $10 \mu \mathrm{m}^{-1}$.

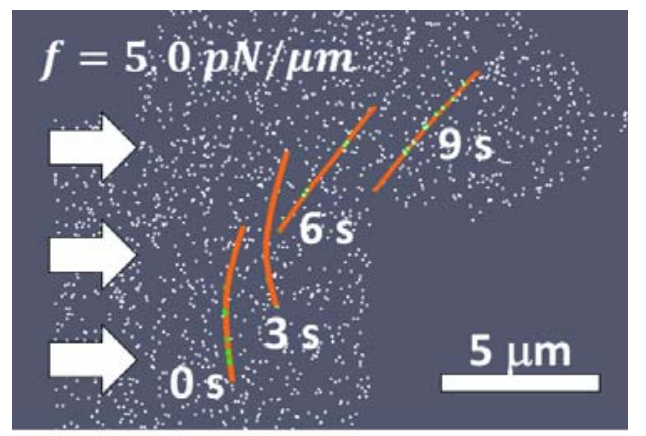

Figure 2. A representative gliding movement of microtubule under a uniform external force. The orange lines represent microtubules at 0, 3, 6 and $9 \mathrm{~s}$. Green dots represent kinesins attaching to the microtubules. White dots represent kinesins. The external force was applied from the right. The surface density of kinesins was $10 \mu \mathrm{m}^{-1}$.

When the force density was increased, the microtubules showed sidewise slipping and were eventually dissociated from the substrate. Figure 2 shows a representative gliding movement of a microtubule. The surface density of kinesin was $10 \mu \mathrm{m}^{-2}$. The force density was $5.0 \mathrm{pN} / \mu \mathrm{m}$. The microtubule did not follow its leading tip. The gliding movement was not smooth and interrupted by sidewise slippings. After slipping sideways, the microtubule was dissociated. The sidewise slipping [9] and dissociation [5] due to external force have been observed in experiments. Our simulation showed qualitative agreements with them.

\section{SUMMARY}

In this study, we have extended our previous work to include gliding movements of microtubules under external force. The simulation results were qualitatively in agreement with experimental ones.

However, further investigations are clearly needed. In particular, more realistic force fields are required for quantitative comparisons with experiments. Once such improvements have been done, this simulation would enable better understanding of details of the gliding movements of microtubules under external force.

\section{ACKNOWLEDGMENTS}

This work was partially supported by JSPS Grant-in-Aid for Young Scientists (B) under Grant 24760203.

\section{REFERENCES}

[1] Hess, H., Engineering Applications of Biomolecular Motors. Annual Review of Biomedical Engineering, 2011. 13: p. 429-450.

[2] Nakano, T., et al., Molecular Communication and Networking: Opportunities and Challenges. NanoBioscience, IEEE Transactions on, 2012. 11(2): p. 135-148.

[3] Atakan, B., Active Molecular Communication, in Molecular Communications and Nanonetworks2014, Springer New York. p. 145-179.

[4] Farsad, N., et al., A Comprehensive Survey of Recent Advancements in Molecular Communication. arXiv preprint arXiv:1410.4258, 2014.

[5] Agayan, R.R., et al., Optimization of Isopolar Microtubule Arrays. Langmuir, 2013. 29(7): p. 2265-2272.

[6] Nitta, T., A. Tanahashi, and M. Hirano, In silico design and testing of guiding tracks for molecular shuttles powered by kinesin motors. Lab On A Chip, 2010. 10(11): p. 1447-1453.

[7] Ishigure, Y. and T. Nitta, Understanding the Guiding of Kinesin/Microtubule-Based Microtransporters in Microfabricated Tracks. Langmuir, 2014. 30(40): p. 1208912096.

[8] Ishigure, Y. and T. Nitta, Simulating an Actomyosin in Vitro Motility Assay: Toward the Rational Design of ActomyosinBased Microtransporters. NanoBioscience, IEEE Transactions on, 2015. 14(6): p. 641-648.

[9] van den Heuvel, M.G., M.P. de Graaff, and C. Dekker, Molecular sorting by electrical steering of microtubules in kinesin-coated channels. Science, 2006. 312(5775): p. 910-4.

[10] Isozaki, N., et al., Control of microtubule trajectory within an electric field by altering surface charge density. Scientific Reports, 2015. 5: p. 7669. 